\title{
Self-encoded Marker for Optical Prospective Head Motion Correction in MRI ${ }^{\star}$
}

\author{
Christoph Forman ${ }^{1,2}$, Murat Aksoy ${ }^{1}$, \\ Joachim Hornegger ${ }^{2}$, and Roland Bammer ${ }^{1}$ \\ 1 Department of Radiology, Stanford University, Stanford, California, USA \\ 2 Pattern Recognition Lab, Friedrich-Alexander-University Erlangen-Nuremberg, \\ Erlangen, Germany
}

\begin{abstract}
The tracking and compensation of patient motion during a magnetic resonance imaging (MRI) acqusition is an unsolved problem. For brain MRI, a promising approach recently suggested is to track the patient using an in-bore camera and a checkerboard marker attached to the patient's forehead. However, the possible tracking range of the head pose is limited by the locally attached marker that must be entirely visible inside the camera's narrow field of view (FOV). To overcome this shortcoming, we developed a novel self-encoded marker where each feature on the pattern is augmented with a 2-D barcode. Hence, the marker can be tracked even if it is not completely visible in the camera image. Furthermore, it offers considerable advantages over the checkerboard marker in terms of processing speed, since it makes the correspondence search of feature points and marker-model coordinates, which are required for the pose estimation, redundant. The motion correction with the novel self-encoded marker recovered a rotation of $18^{\circ}$ around the principal axis of the cylindrical phantom in-between two scans. After rigid registration of the resulting volumes, we measured a maximal error of $0.39 \mathrm{~mm}$ and $0.15^{\circ}$ in translation and rotation, respectively. In in-vivo experiments, the motion compensated images in scans with large motion during data acquisition indicate a correlation of 0.982 compared to a corresponding motion-free reference.
\end{abstract}

\section{Introduction}

Patient motion during data acquisition remains a challenging problem in MRI. The consequences are often significant image artifacts which lower the diagnostical confidence of the image data. Recent publications have proposed methods to reduce or compensate the impact of motion on the images. Techniques using PROPELLER or spiral sequences correct patient motion with alternative data acquisition strategies 122. Navigator echos are added to MR sequences

\footnotetext{
* This work was supported in part by the National Institutes of Health (Grant numbers: 1R01EB008706, 5R01EB002711, 1R01EB006526, 1R21EB006860, P41RR09784), Lucas Foundation, Oak Foundation, Bavarian California Technology Center, and GE Healthcare.
} 
to compensate retrospectively and prospectively patient's motion during the scan [345]. Another MR based motion correction method was introduced by Ooi et al. 6], using the response of active markers in form of small coils attached to the forehead of the patient. For a data acquisition independent approach, external tracking systems were proposed. In order to transfer the detected motion of these systems to motion, which actually occurred in the scanner image plane, a cross-calibration of both frame of references is required. External optical systems outside the scanner bore were used to track a marker attached to the patient's head [7. Drawback of this system is, that it requires a line of sight on the marker inside the scanner. Aksoy et al. 8] introduced a motion correction system with an in-bore camera. In this approach, an MR-compatible camera is mounted on the head coil, tracking the position and orientation of a checkerboard marker attached to the patients forehead. One essential constraint of this method is that once the marker is occluded by another object or is partly outside the camera's field of view (FOV), no motion correction is possible anymore. The restricted space inside the scanner bore entails camera-marker distances between 5 and $7 \mathrm{~cm}$. Additionally, the shape of different coils may occlude parts of the camera FOV. Thus, the restriction of the marker detection delimits the possible tracking range of the patient's head position. To overcome this limitation, we developed the self-encoded marker with additional codes for each feature point.

\section{Materials and Methods}

The motion correction system was implemented on a GE Signa 1.5T whole body system (GE Healthcare, Milwaukee, WI). Fig. 1 shows the setup of the system. The MR-compatible camera is mounted on the 8 channel head coil. Infrared diodes attached to the camera body illuminate the scene inside the scanner bore. An independent tracking computer processes the captured camera images as described in 89 . The patient's pose at the beginning of each scan serves as initial point of reference to describe the motion throughout the scan. For each camera image, the detected motion of the optical system is transformed into motion, which actually occurred in the scanner image plane. This requires an initial cross-calibration of the tracking system with the MR scanner. The pose updates in form of translation and rotation are relative to the patient's initial position at the first data acquisition. They are sent in real-time via network connection to the MR sequencer. Assuming rigid head motion, these updates are directly used by the sequencer to adjusts the gradients and radio frequencies before each data acquisition. That way, the slice position and orientation is determined by scanned anatomy and not by the scanner geometry. Once a large difference in rotation or translation between two subsequent pose updates was detected, the current acquisition data was disregarded and repeated to compensate the latency of the entire scan.

Basis of the self-encoded marker is a checkerboard pattern. Adjacent corners of neighboring quads on this pattern describe the feature points of this marker. For the checkerboard marker all features are required to establish the point correspondences of detected feature points in the camera image and their model 


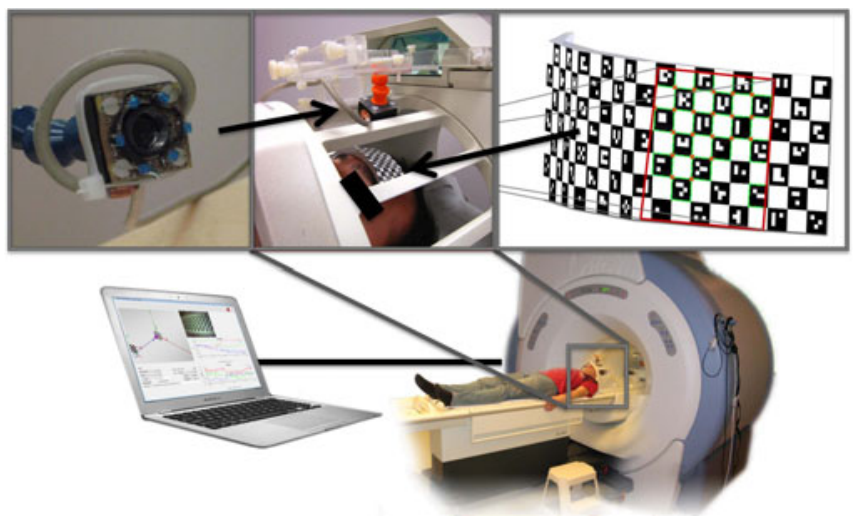

Fig. 1. Setup of the optical motion correction system: The MR compatible camera is mounted on the 8 channel head coil. The signal is processed by an external tracking computer, which sends the pose updates to the MR scanner via network connection.

coordinates in the marker-model geometry. Within the black quads of the selfencoded marker, 2-D barcodes similar to the ARTag marker [10] are embedded. These unique codes identify each feature of the pattern, specify its position on the marker geometry and consequently define the aforementioned point correspondences. In contrast to the ARTag marker we are using a 10 bit encoding instead of 36 bit for the embedded code. The redundancy in the ARTag marker permits a verification of the code. Instead of verifying each quad independently, we compare the recognized code of each quad and its neighboring quads with a map containing all positions of the codes on the marker. That way, every bit of the embedded code can be used for the encoding, which leads to a more robust detection of the marker in the in-bore camera image. Based on the defined point correspondences and the known intrinsic camera parameters, the marker pose is estimated by homography (planar marker) or direct linear transformation (3-D marker). Even if only parts of the self-encoded marker are visible to the camera, its pose can still be determined. Furthermore, different feature points of the selfencoded marker can be used for a robust tracking of the marker position and orientation. That way, we overcome the limitation of the checkerboard marker to the camera's FOV.

For the pose estimation of the marker, first, the captured camera image is converted into a binary image by thresholding. The outline of the black quads is detected by quadringular contours in this image. Using the boundary of each quad we sample the interior into a $5 \times 5$ grid. Then, the embedded code in the inner $3 \times 3$ cells is classified by thresholding into a binary code. By means of a-priori knowledge of the marker layout, the recognized codes are verified as mentioned above. That way, erroneous detected quads can be eliminated. Finally, the relative pose of the marker to the camera is estimated using the point correspondences of detected features and marker-model points. 


\section{Experiments and Results}

Phantom and in-vivo experiments were performed using an axial 3D spoiled gradient recalled $\left(\mathrm{SPGR}\right.$ ) sequence with $\mathrm{TR}=9.5 \mathrm{~ms}, \mathrm{TE}=4.1 \mathrm{~ms}$, flip angle $=20^{\circ}$, slice thickness $=1.5 \mathrm{~mm}, \mathrm{FOV}=24 \mathrm{~cm}$, and a resolution of $192 \times 192 \times 96$.

\subsection{Phantom Experiment}

We evaluated the accuracy of the optical motion correction system with a cylindrical phantom and two subsequent MRI scans. In-between both scans, the static phantom was manually rotated about its principal axis by $18^{\circ}$. The first scan used as a reference, was compared to the motion corrected second scan. Assuming an ideal motion correction system, we expected an identical image of the phantom in both scans. For qualitative evaluation, Fig. 2 shows both scans and the difference image. We performed this experiment with both markers. The difference images indicate a discrepancy of the structure at the top of the phantom, which is caused by the phantom being not completely filled with water. While the structure of the phantom was rotated, the water remained at the same position. For quantitative evaluation of the residual mismatch, retrospective rigid registration was performed. This registration resulted in a remaining offset of:

$$
\begin{aligned}
& \boldsymbol{t}_{\text {self-encoded }}=\left(\begin{array}{lll}
-0.36, & 0.10,-0.39
\end{array}\right) \quad[\mathrm{mm}] \\
& \boldsymbol{r}_{\text {self-encoded }}=\left(\begin{array}{lll}
0.11, & 0.00, & 0.15
\end{array}\right) \quad\left[^{\circ}\right] \\
& \boldsymbol{t}_{\text {checkerboard }}=\left(\begin{array}{ll}
0.89, & 0.09,-0.79
\end{array}\right) \quad[\mathrm{mm}] \\
& \boldsymbol{r}_{\text {checkerboard }}=(-0.35,-0.03,-0.29) \quad\left[{ }^{\circ}\right]
\end{aligned}
$$

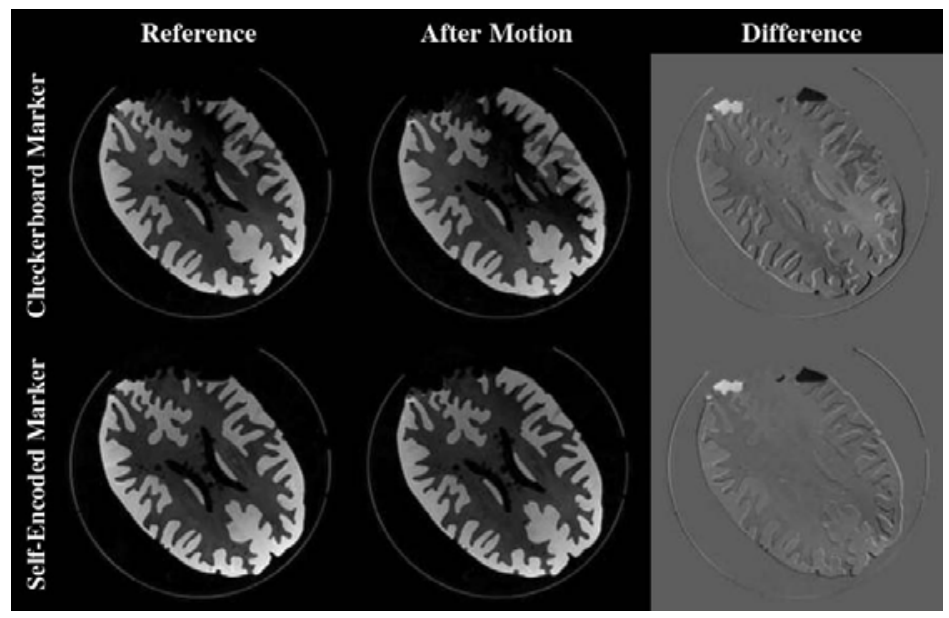

Fig. 2. The reference scan is compared to a scan with correction after $18^{\circ}$ rotation. Both difference images are with a contrast enhancement by a scaling factor of 2 . 


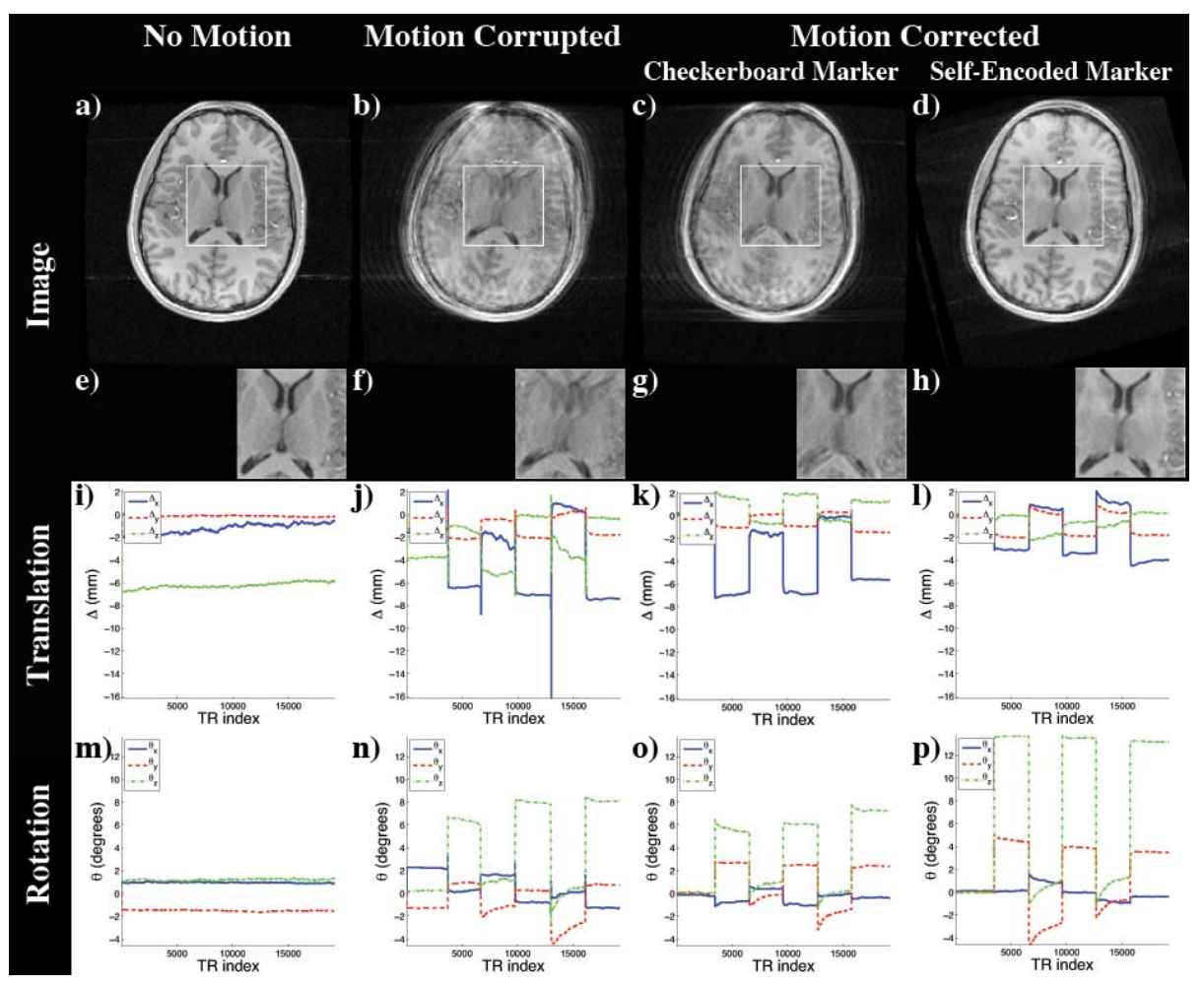

Fig. 3. Images of the scan comparing self-encoded and checkerboard marker: (a) Reference Scan; Scan with motion and (b) no correction, motion correction using the pose updates of the (c) checkerboard and the (d) self-encoded marker; (e-h) Magnification of window in (a-d); Detected translation (i-l) and rotation (m-p)

\subsection{In-vivo Experiments}

In-vivo experiments were performed on a healthy volunteer to evaluate the position estimates of the self-encoded marker for motion correction. For each scan, the obtained pose estimates relative to the initial head position were recorded in a $\log$ file.

In the first experiment, the pose estimates of the reference checkerboard marker and the novel self-encoded marker were compared. Four scans were obtained for this experiment. In order to track the head motion during data acquisition, first the checkerboard marker was attached to the forehead. In the first scan, the volunteer was instructed to maintain a stationary head position to create a motion-free reference image. For the following scans, the subject was asked to perform a similar head rotation every 30 seconds in order to assure a comparable motion pattern. In the second scan, the motion-correction system was turned off and the position estimates of the checkerboard marker were recorded. The obtained pose updates of this marker were used in the third scan to adapt 


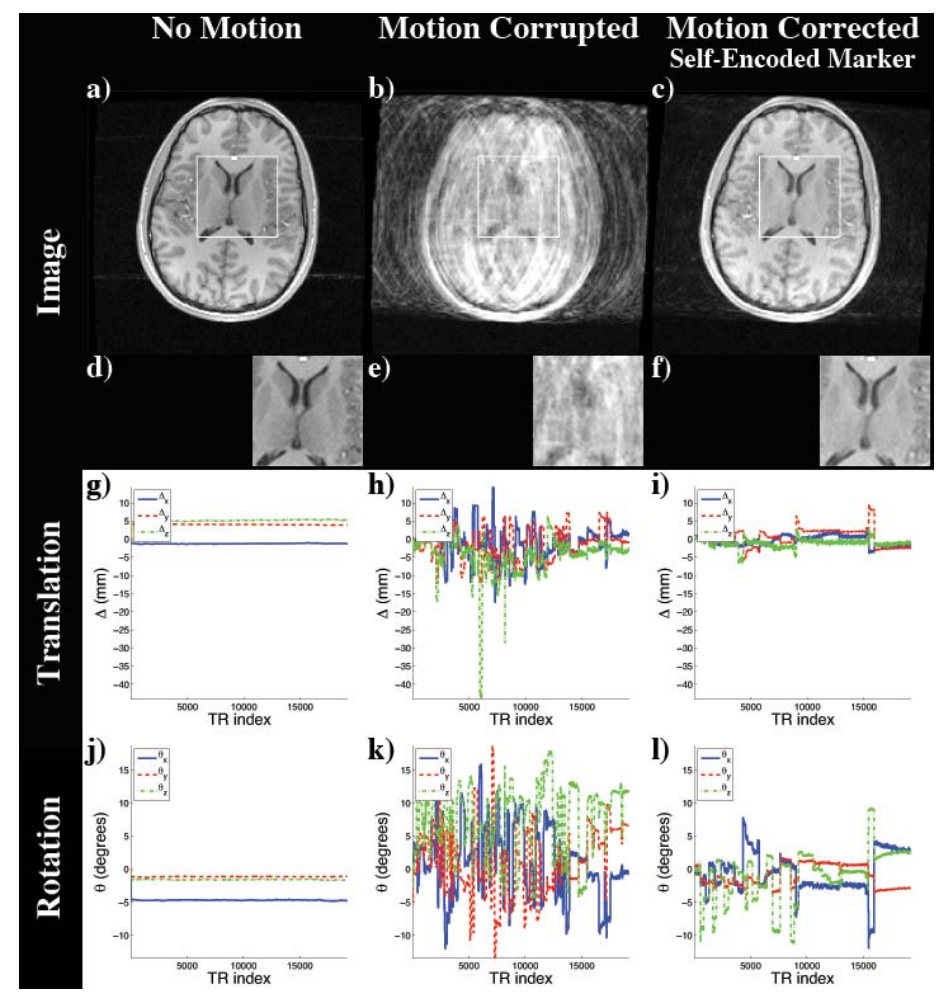

Fig. 4. Images of the scan simulating an uncooperative patient: (a) Reference Scan; Scan with random motion and (b) no correction and (c) prospective motion correction using the self-encoded marker; (d-f) Magnification of window in (a-c); Detected translation $(\mathbf{g}-\mathbf{i})$ and rotation $(\mathbf{j}-\mathbf{p})$

the scanner for motion, while in the last scan the self-encoded marker was attached to the forehead to track the volunteers head motion. Fig. 3 shows the resulting images of the performed scans. Without correction, the MRI images exhibited motion artifacts. Using the pose updates of the checkerboard marker, these artifacts were reduced. However, inaccuracies of the marker became apparent in a mismatch of the scanned anatomical structure. In this camera setup the tracking range of the checkerboard marker was restricted to $6^{\circ}$. The self-encoded marker was able to extend it to a head rotation of $13^{\circ}$, which is maximal without touching the coil. The improvement in accuracy of the self-encoded marker compared to the checkerboard marker was measured by Pearson's correlation coefficient [11]. Whereas the correlation of reference and motion-corrupted image resulted in a coefficient of 0.908 , the optical tracking system using the pose updates of the checkerboard marker improved this value to 0.936 . Using the self-encoded marker for the tracking of the volunteer showed a correlation of 0.971 . 
In the second experiment, the volunteer was asked to simulate an uncooperative behavior. First, a reference image was acquired. Then, in the following scans, the volunteer performed a random trembling motion for the entire scan. The pose estimates of the self-encoded marker were used to describe the head position over time during the data acquisitions. For the second scan, the detected translation and rotation were recorded whereas the scanner was also adapting for motion in the third scan. Due to the performed random motion, it was not possible to repeat the experiment with the identical motion pattern. Continuous motion has a strong impact on the resulting images, see Fig. 4. The motion induced artifacts corrupted the entire anatomical structure of the brain. While adapting the scanner geometry based on the detected head pose of the volunteer, the system was able to recover the structure of the brain. The effects of motion resulted in a correlation coefficient of 0.858 , while the pose updates of self-encoded marker were able to improve this factor to 0.982 .

\subsection{Performance}

We compared the performance of the self-encoded marker with 76 features to a checkerboard marker with 20 features. The captured image of the in-bore camera had a resolution of $640 \times 480$ pixel. For the analysis of the computational time the software run on a Intel Core2Duo CPU $(2.26 \mathrm{GHz})$. The entire process of feature detection and point correspondence search took $17.2 \mathrm{~ms}$ for the selfencoded marker and $28.6 \mathrm{~ms}$ for the checkerboard marker (acceleration factor $1.7 \mathrm{x})$. Compared to a common checkerboard detection algorithm, this factor was achieved with a optimized detection of the self-encoded marker, since its outcome is not crucial for the generation of the point correspondences.

\section{Discussion}

A crucial limitation of existing in-bore tracking systems for prospective motion correction in MRI is the narrow FOV of the camera. In this study, we introduced a novel marker design with embedded 2-D barcodes that identify each feature on the pattern. Recognizing these codes in the captured camera image the tracking algorithm is able to estimate the pose of the self-encoded marker in situations where the marker is only partly visible.

We compared the accuracy of the self-encoded and checkerboard marker in a phantom experiment. The rotation of the phantom in-between two scans was compensated by the motion correction system in the second scan. Both resulting MR images were rigidly registered, which showed an improved accuracy for the self-encoded marker with a maximal offset of $0.39 \mathrm{~mm}$ and $0.15^{\circ}$ for translation and rotation, respectively. In the first in-vivo experiment, we compared the pose estimates of both markers for motion correction. This comparison study indicated the restricted range of motion that can be tracked with the checkerboard marker. The tracking range was extended from $6^{\circ}$ with the checkerboard marker to $13^{\circ}$ using the self-encoded marker. Furthermore, replacing the checkerboard 
marker with the self-encoded marker increased the correlation of the resulting motion compensated MR images from 0.936 to 0.971 compared to a motion-free reference. In case of an uncooperative patient the motion compensation based on the pose updates of the self-encoded marker was able to recover the scanned anatomical structure. The correlation of the motion compensated scan resulted in a coefficient of 0.982 compared to a reference scan without motion.

In order to provide an estimate of the patient's head position for every data acquisition step, the total latency of the prospective motion correction system must not exceed the repetition time. Although we were able to accelerate the processing time of the self-encoded marker by a factor of 1.7 , there is still room for further improvements.

\section{References}

1. Pipe, J.G.: Motion Correction With PROPELLER MRI: Application to Head Motion and Free-Breathing Cardiac Imaging. Magn. Reson. Med. 42(5), 963-969 (1999)

2. Sarty, G.E.: Single TrAjectory Radial (STAR) imaging. Magn. Reson. Med. 51(3), 445-451 (2004)

3. Thesen, S., Heid, O., Mueller, E., Schad, L.R.: Prospective Acquisition Correction for Head Motion With Image-Based Tracking for Real-Time fMRI. Magn. Reson. Med. 44(3), 457-465 (2000)

4. Aksoy, M., Liu, C., Moseley, M., Bammer, R.: A Self-navigated Spiral In \& Out Pulse Sequence Design for Retrospective Motion Correction. In: Proceedings of the 14th Annual Meeting of the ISMRM, Seattle, Washington (2006)

5. White, N., Roddey, C., Shankaranarayanan, A., Han, E., Rettmann, D., Santos, J., Kuperman, K., Dale, A.M.: PROMO: Real-Time Prospective Motion Correction in MRI Using Image-Based Tracking. Magn. Reson. Med. 63(1), 91-105 (2010)

6. Ooi, M.B., Krueger, S., Thomas, W.J., Swaminathan, S.V., Brown, T.R.: Prospective real-time correction for arbitrary head motion using active markers. Magn. Reson. Med. 62(4), 943-954 (2009)

7. Dold, C., Zaitsev, M., Speck, O., Firle, E.A., Hennig, J., Sakas, G.: Prospective Head Motion Compensation for MRI by Updating the Gradients and Radio Frequency During Data Acquisition. In: Duncan, J.S., Gerig, G. (eds.) MICCAI 2005. LNCS, vol. 3749, pp. 482-489. Springer, Heidelberg (2005)

8. Aksoy, M., Newbould, R., Straka, M., Holdsworth, S., Skare, S., Santos, J., Bammer, R.: A Real Time Optical Motion Correction System Using a Single Camera and 2D Marker. In: Proceedings of the 16th Annual Meeting of the ISMRM, Toronto, Canada (2008)

9. Zaitsev, M., Dold, C., Sakas, G., Hennig, J., Speck, O.: Magnetic Resonance Imaging of Freely Moving Objects: Prospective Real-time Motion Correction Using an External Optical Motion Tracking System. NeuroImage 31(3), 1038-1050 (2006)

10. Fiala, M.: ARTag, a Fiducial Marker System Using Digital Techniques. In: Proceedings of the IEEE Computer Society Conference on Computer Vision and Pattern Recognition (CVPR), pp. 590-596 (2005)

11. Edwards, A.L.: An Introduction to Linear Regression and Correlation. W. H. Freeman and Co., San Francisco (1993) 